\title{
Patients' perspective in postoperative pain management: role of Sufentanil Sublingual Tablet System in major orthopedic surgery
}

Coluzzi $F^{1}$, Bramato $A^{1}$, Scerpa $M^{1}$, Corona $A^{1}$, Favetti $F^{1}$, De Martino $E^{1}$, Celidonio $L^{1}$, Locarini $P^{1}$ ${ }^{1}$ Unit of Anesthesia, Intensive Care and Pain Therapy Dept. Medical and Surgical Sciences and Biotechnologies SAPIENZA University of Rome, Polo Pontino - Latina, Italy

\section{Background:}

Inadequate post-operative main management is one of the most common complain after major orthopedic surgery [1]. According to guidelines, intravenous patient-controlled analgesia (IV-PCA) is one of the proposed analgesic techniques. Limits of iv-PCA have been identified in programming errors, invasive access, impairment of mobility, and patients' errors. The sufentanil sublingual tablet system Zalviso ${ }^{\circledR}$ (SSTS) is a non-invasive, easy-to-use and effective innovative PCA, which has been shown to be an effective alternative to traditional parenteral analgesia [2].

\section{Methods:}

Adults, who had undergone major orthopedic surgery, with expected postoperative pain $\geq 4$ on a 11-point numeric rating scale, received SSTS $15 \mathrm{mcg}$, with a 20-minute lockout interval, as requested for pain, over the 72-hour study period. Data were collected at baseline (T0) and 3, 6, 12, 24, 36, 48, 60, 72 hours after surgery. The primary endpoint was the Patient Global Assessment (PGA) on a 5-point categorical scale (excellent, very good, good, fair, poor). Secondary endpoints were analgesic efficacy, need for supplemental rescue doses, occurrence of side effects and sleep pain interference.

\section{Results:}

36 patients were enrolled. Average age was 67.4 years (SD 10.5 years), with $20 \%$ being $>65$ years. $97.2 \%$ of patients evaluated the treatment as excellent or very good on PGA (Fig.1). No patients discontinued due to inadequate analgesia or adverse events. Average baseline pain intensity was 2.4 (SD 2.2) and it remained $<3$ along all the study period (Fig.2).

\section{Fig1: Patients' and Caregivers' Global Assessment}

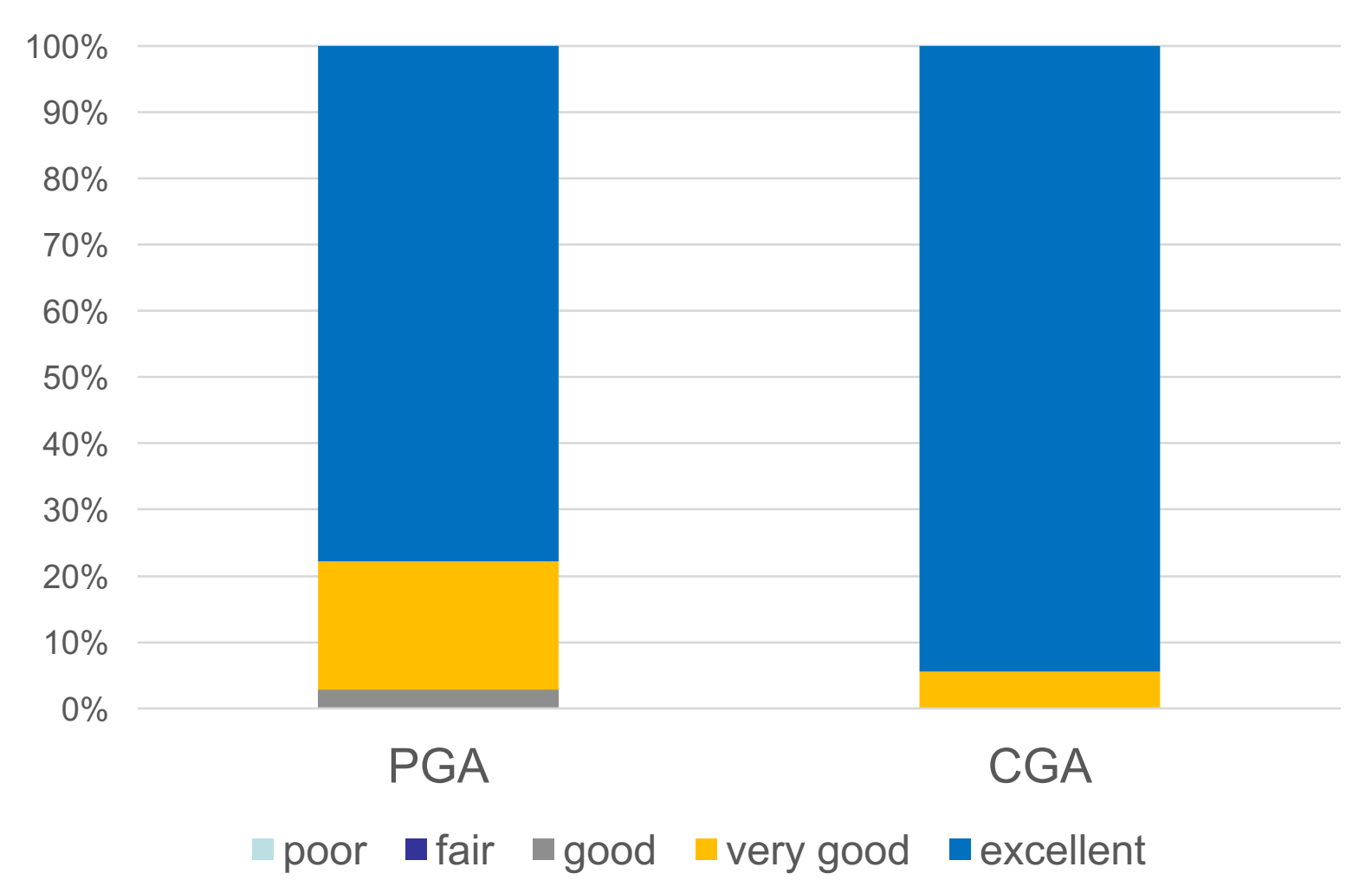

Fig 2: Pain Intensity in the first 72 post-operative hours

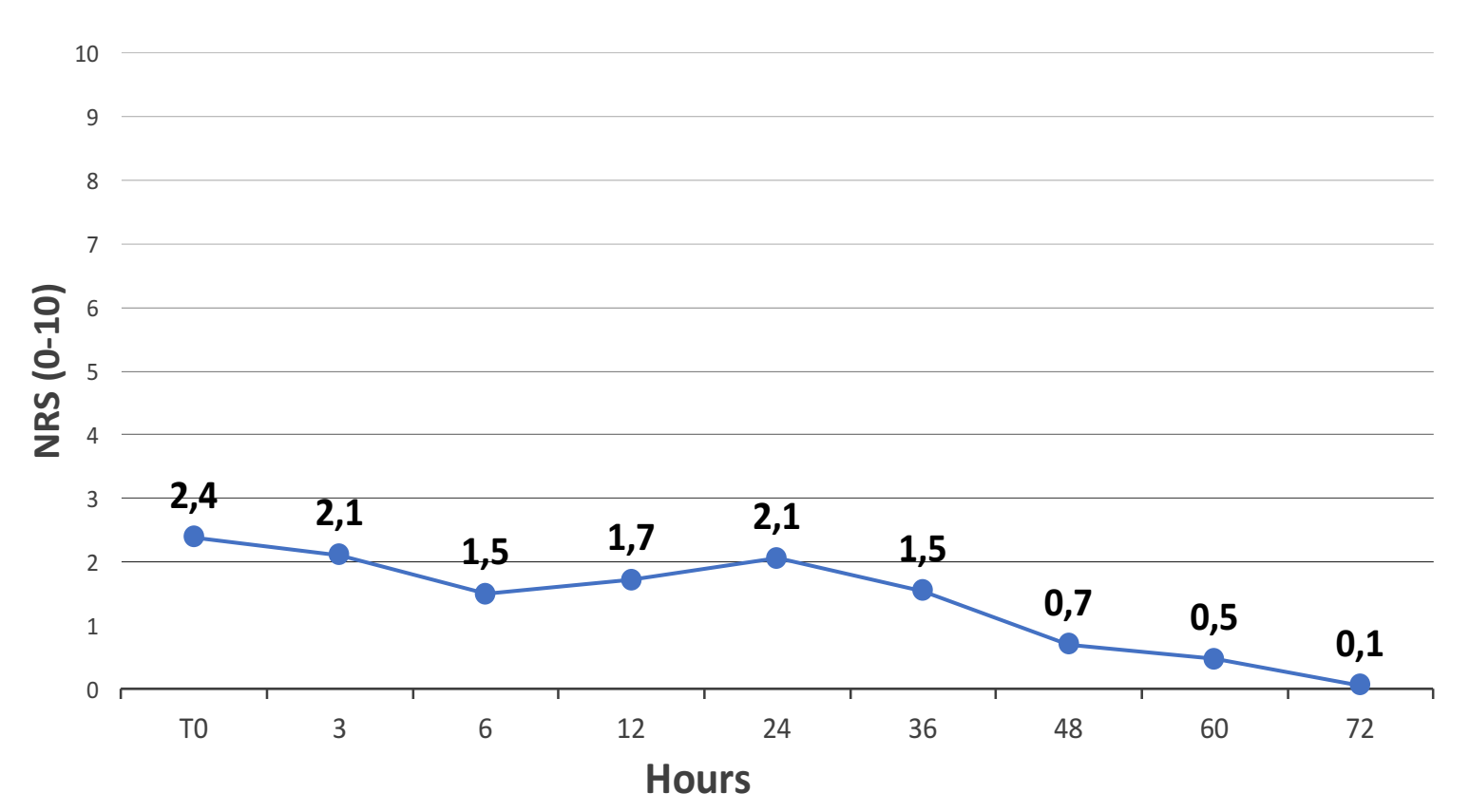

Required SSTS doses ranged from 5 to 48 in 72 hours. The most common surgeries were total hip arthroplasty (THA, $47.2 \%$ ) and total knee arthroplasty (TKA, 33.3\%), followed by major spine surgery $(11.1 \%)$ and shoulder arthroplasty (SA, 8.3\%). SSTS doses were significantly higher in TKA (29.8), compared with THA (22), SA (17), and spine surgery (12.3). Mean required SSTS doses in different surgeries are shown in (Fig. 3). Timing for the first SSTS dose was longer in patients undergoing single-shot peripheral nerve block (216.8 min) compared with those receiving general or spinal anesthesia (69.6 and 74.5 min respectively). Rescue doses were needed in $5.5 \%$ of SSTS patients. Quality of sleep was good or very good in $86.1 \%$ of patients on the same day of surgery.

\section{Conclusions:}

SSTS was well accepted by patients undergoing major orthopedic surgery and resulted in effective management of moderate-to-severe acute postoperative pain.

\section{Fig 3: SSTS doses in major orthopedic surgery}

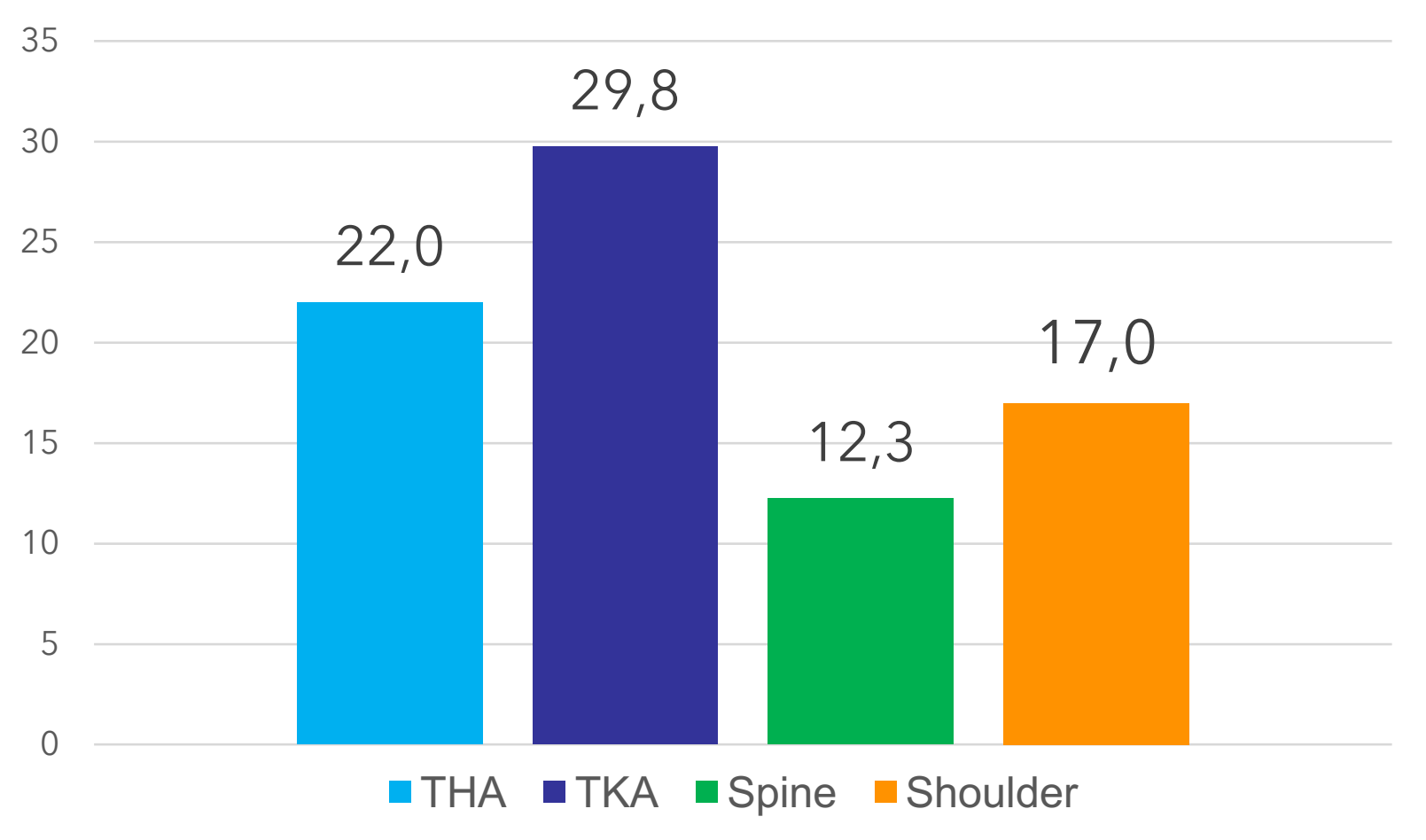

